\title{
Derivati dei nomi dei mesi (Settembre)
}

\author{
Lucia Francalanci
}

PUBBLICATO: 26 SETTEMBRE 2021

\section{Quesito:}

Dopo le schede dedicate ai derivati digennaio, febbraio, marzo, aprile, maggio, giugno, luglio e agosto, continuiamo la pubblicazione della nostra rubrica con la scheda relativa a settembre. Per le informazioni generali e la bibliografia si rimanda all'Introduzione.

\section{Derivati dei nomi dei mesi (Settembre)}

I 1 termine settembre deriva dal latino septèmber -bris (mensis), derivato di septem 'sette', perché era il settimo mese dell'anno nel calendario romano. Nonostante settembre sia per la gran parte un mese estivo, dato che l'inizio dell'autunno coincide con quello della sua ultima decade, è spesso associato alla stagione autunnale; questo legame ci viene ad esempio suggerito da alcuni sostantivi di uso antico o letterario derivati dal nome del mese e che designano proprio l'autunno: settembreccia, settembresca, settembria. Nel linguaggio giornalistico e storiografico il nome settembre è inoltre legato alle stragi (settembrate) compiute dai rivoluzionari (detti settembrizzatori, settembristi o settembriani) durante la rivoluzione francese.

Tra i modi di dire legati al sostantivo, si ricordano le espressioni rimandare a settembre (nel passato ordinamento scolastico, far sostenere a uno studente esami di riparazione nella sessione cosiddetta autunnale) e esami di settembre (esami di riparazione che gli studenti rimandati devono sostenere nella stessa sessione) e la locuzione Settembre nero (nome dell'organizzazione terroristica palestinese costituita nel settembre I970 e attiva fino al I973, che prende il nome dalla dura repressione compiuta nel settembre del 1970 da re Hussein di Giordania contro i palestinesi; l'espressione viene usata a volte sulla stampa anche per riferirsi a situazioni difficili, relative all'economia, al clima, alla politica, previste dopo la conclusione delle vacanze estive).

- settembrale

Registrato esclusivamente dal DELI, dal GDLI e dal GRADIT, settembrale è un aggettivo di uso letterario che sta per 'di settembre, settembrino'. Si trova, ad esempio, in D’Annunzio:

Sotto il ciel settembrale / che riversa il suo calice d'oro / ampio dal Celio al Viminale / dal Gianicolo al Vaticano / dall'Anfiteatro al Foro / nel di fausto dell'alta conquista, / cantiamo l'avvento fatale. (Gabriele D’Annunzio, Versi d'amore e di gloria, vol. II, Milano, Mondadori, I984, p. 279)

- settembrata

Il sostantivo settembrata non è registrato dai dizionari sincronici o storici, ma risulta ben attestato sia in rete sia nei volumi a stampa. Quanto al significato, il termine presenta diverse accezioni. Può indicare una festa popolare (specialmente una festa di partito, come la festa dell'Unità) o un ritrovo festoso tra amici:

Prima di entrare a lavorare vi credevo alla loro utilità, e militavo attivamente nel partito operaio cioè 
PCI, oggi sono solo tesserato e non partecipo quasi mai alla vita del partito, vado soltanto ad aiutare quando si fa la festa della settembrata, un po' lì nei banchi a controllare quando c'è confusione. (Vincenzo Guerrazzi, L'altra cultura: inchiesta operaia, Padova, Marsilio Editori, 1975, p. 245)

Sarà una staffetta lunga sei giorni, la settembrata di quest'anno. E avrà due sedi: il 6, 7 e 8 settembre comincerà in Val Bisagno, a San Fruttuoso, in piazza Martinez. Proseguirà poi, postandosi a Ponente, il 20, 2г e 22 settembre, a Prà, in piazza Sciesa. (Michela Bompan, La Festa dell'Unità raddoppia per ripartire lancia la periferia, "la Repubblica", 4/8/2019)

Vieni. Domenica sera si farà una porchetta squisita con accompagnamento di maccaroni co ' $i$ 'vongole, in casa di Ciccutiello. Non devi mancare. È l'ultima settembrata e non devi mancare. Ti dirò a voce quel che non ho potuto scriverti. Perdona il silenzio, interpretato male da te. Ti abbraccio. Vieni. Ti vogliamo. Tuo Gabriele (D'Annunzio e Filippo De Titta: carteggio (1880-1922) e altri documenti dannunziani, a cura di Enrico di Carlo, Lanciano, Carabba, 2007, p. I23)

Nel contesto della rivoluzione francese, invece, si riferisce ai massacri di settembre, episodio della rivoluzione segnato dall'esecuzione di oltre mille prigionieri che ebbe luogo nelle carceri di Parigi dal 2 al 6 settembre 1792 :

Tutti i giorni da che si formò, dalla sua ringhiera altro non si sentivano che le più anarchiche dottrine, e le più incendiarie proposte; insurrezione, strage e settembrate, erano sempre in tabella per discutersi e decretarsi! (Louis Vivien, Storia generale della rivoluzione francese, dellimpero, della restaurazione, e della monarchia del 1830, fino al 1841, tomo II, vol. II, Firenze, Giuseppe Celli, I849, p. 292)

Tutti i tumulti, gli spogliamenti, gl'incendi, le carceri, le mitragliate, le settembrate, le ghigliottine della rivoluzione francese donde sbucarono? Dall'esautoramento di Luigi XVI. (AA.VV., Dei doveri del popolo. Quando il sovrano é calunniato, "La civiltà cattolica", serie IV, vol. V, Roma, I860, p. 46I)

\section{- settembreccia/settembresca/settembria}

I sostantivi settembreccia e settembresca sono antichi termini per designare l'autunno, registrati esclusivamente dai dizionari storici (GDLI, Tommaseo-Bellini, I-IV edizione del Vocabolario degli accademici della Crusca), probabilmente perché ormai da tempo in disuso. Gli esempi forniti sono gli stessi in tutti i dizionari e sono tratti dal Libro della sanitá del corpo di Aldobrandino da Siena, volgarizzamento de Le régime du corps (а. ІзІо):

Se la settembreccia tien della natura della state siccome di caldo, sì val meglio a darla di notte.

Lo malinconoso è freddo e secco, che' sembra vile alla settembreccia [dal GDLI: sembrare vile alla settembreccia $=$ essere più triste dell'autunno].

Sappiate che queste pistolenze avvengono più nella settembresca, che nelle altre stagioni dell'anno.

La voce settembria, sinonimo delle precedenti, è invece presente anche in alcuni dizionari sincronici (Zingarelli e GRADIT), che la datano anteriormente al I348 e la marcano come termine raro o obsoleto:

Gli ambasciadori del re d'Inghilterra promisono per lo loro re gaggi e soldi agli Alamanni e agli altri allegati, e la venuta del re in persona... alla settembria. (Giovanni Villani, Cronica, a cura di Ignazio Moutier e Francesco Gherardi Dragomanni, Firenze, Coen, I844-45, p. 72) 
Dopo gli acquazzoni estivi gli acini dell'uva sogliono imbrunire e saracinare sotto l'occhio del sole e ai molli tepori della settembria. (Vincenzo Gioberti, Della protologia, a cura di Giuseppe Massari, vol. II, Torino, Eredi Botta, I857, p. 510)

\section{- settembresco}

L'aggettivo settembresco non è registrato dai dizionari ma se ne trovano diverse attestazioni in riferimento alle già citate stragi di settembre, avvenute nel contesto della rivoluzione francese:

Le vittime fecersi adesso carnefici, e i medesimi orrori che inaugurarono il regno di Robespierre distinsero in queste infelici contrade l'avvenimento della nuova potestà, mentre i trucidamenti della ghiacciaja e i massacri settembreschi tornarono quivi a farsi vedere. (Louis Vivien, Storia generale della rivoluzione francese, dellimpero, della restaurazione, e della monarchia del 1830, fino al 1841, tomo III, Firenze, Giuseppe Celli, I843, p.I28)

\section{- settembriano/settembrista/settembrizzamento/settembrizzare/settembrizzatore}

Il sostantivo settembrizzatore, registrato da tutti i dizionari, non è un derivato di settembre, ma probabilmente un adattamento del francese septembriseur (derivato di septembre 'settembre'), termine giornalistico e storiografico coniato in Francia nel 1793 (come ci dice il Trésor de la langue française), ed entrato poi in italiano, per indicare 'chi, durante la rivoluzione francese, prese parte ai massacri di settembre' e, per estensione, 'chi compie crudeli e spietati delitti politici'.

Sarà bene mettere sotto gli occhi de' lettori due pagine della storia della rivoluzione francese scritta da Giulio Michelet e le orribili facce dei settembrizzatori. (Giosue Carducci, Opere, vol. XXIV, Bologna, Zanichelli, I950, p. 4I2)

Il GDLI registra anche l'uso figurato 'critico eccessivamente severo o malevole stroncatore'.

I dizionari italiani riportano il 1799 come data della prima attestazione del sostantivo settembrizzatore, ma, tramite Google libri, è possibile rintracciarne un'occorrenza già nel I793:
Brissot, uno di quegli che smascherarono con maggiore energia i mostri a' quali Anacharsis Clootz dette il nome di Settembrizzatori, riporta nel Patriota Francese che dirigeva, che essendosi portato il 4 settembre presso il ministro della giustizia per esprimergli la sua sorpresa, [...] Danton gli rispose freddamente... (Antonio Fantino Desodoards, Istoria filosofica e imparziale delle revoluzioni di Francia, di Venezia e di Genova, prima traduzione italiana, tomo III, Genova, Stamperia Delle Piane, I793, p. 42)

Il GDLI lemmatizza inoltre il verbo transitivo settembrizzare con la definizione 'massacrare, trucidare (con particolare riferimento ai massacri compiuti dai rivoluzionari francesi nel settembre I792)' e, in senso assoluto, 'compiere stragi'; del verbo settembrizzare vengono riportati anche gli usi figurati di 'distruggere, togliere di mezzo un valore spirituale, la religione' e 'stravolgere'.

'Settembrizzare': fu uno de' primi ornamenti della nuova lingua repubblicana. È termine originalmente francese e significa 'massacrare innocenti, in modo di far inorridir le tigri'. (Lorenzo Ignazio Thjulen, Nuovo vocabolario filosofico democratico, 2 voll., Venezia, Francesco Andreola, 1799, p. 20)

La natura anchessa è dispotica e aristocratica; bisogna democratizzarla (non a caso adopro cotesti vocaboli) senza settembrizzarla. (Niccolò Tommaseo, Del presente e dell'avvenire, a cura di Teresa Lodi, Firenze, Sansoni, 1968, p. I02) 
Il GDLI considera settembrizzare una retroformazione da settembrizzatore, ma non fornisce alcuna data di prima attestazione; in rete possiamo però trovare un'occorrenza del verbo in un volume del I793:

Le prigioni erano oltremodo ripiene, e si parlava di vuotarle con l'eccidio de' carcerati, come s'era eseguito ne' primi giorni di Settembre, dandosi a tale carnificina il grazioso vocalo [sic] di Settembrizzare, come all'appiccare erasi dato quello di lanternare, perché ciò facevasi al ferro di una lanterna. (La storia dell'anno 1793 divisa in sei libri. Parte prima, Venezia, Giuseppe Rossi, I793, p. 48)

È difficile stabilire con esattezza il rapporto derivazionale tra settembrizzatore e settembrizzare, ma dalla ricerca condotta su Google libri sembra che i due termini siano giunti contemporaneamente (nel r793) nella nostra lingua attraverso traduzioni dal francese o cronache della Rivoluzione Francese.

Ancora nel GDLI compare il sostantivo settembrizzamento (nome d'azione da settembrizzare), che indica una 'violenta ondata di reazione contro una violenta ondata rivoluzionaria'.

Vi sono... alcuni mesi celebri per il settembrizzamento dei settembrizzatori. (Lorenzo Ignazio Thjulen, Nuovo vocabolario filosofico democratico, 2 voll., Venezia, Francesco Andreola, I799, p. 2I)

In rete è attestato anche il sostantivo settembrizzazione, con lo stesso significato.

Sinonimi di settembrizzatore sono le voci settembrista e settembriano, derivati direttamente dal nome del mese. Settembrista, presente solo nel GDLI e nel DEI, è considerato una variante storica di settembrizzatore e designa un 'rivoluzionario violento e sanguinario'. Il GDLI registra anche le accezioni 'neofita del nazionalsocialismo giunto al Reichstag con le elezioni del i4 settembre i930' e 'aderente alla costituzione democratica varata in Portogallo il 27 settembre i 822 '.

Dal momento che si vuole enunziarne apertamente il principio e dire che detronizza la religione o che distrugge l'aristocrazia o che stabilisce l'eguaglianza, non s'incontrano che denegazioni, invettive, smentite, e siamo rilegati tra i settembristi, i petrolisti, gli incendiari, gli insensati. (Giuseppe Ferrari, Teoria dei periodi politici, Milano, Hoepli, I874, p. 394)

Nei primi tempi sperò che quel bonapartista, quel giacobino, quel terrorista, quel settembrista ritornasse. Ma passarono le settimane, i mesi, gli anni e, con gran dispiacere del signor Gillenormand, il bevitore di sangue non riapparve. (Victor Hugo, I miserabili, Milano, Rizzoli, 2oII, prima ed. digitale)

Il raro sostantivo settembriano è presente nel GRADIT e nel GDLI come antico sinonimo di settembrizzatore. Il GRADIT riporta il I963 come data della prima attestazione; tale data corrisponde alla pubblicazione dell'articolo Ospizio di parole politiche perdute di Rodolfo de Mattei nella rivista "Lingua nostra" (vol. XXIV, fascicolo 4, dicembre 1963, p. II5), in cui è presente la seguente definizione:

SETTEMBRIANO. Aderente alle famose e sanguinose stragi di aristocratici ed ecclesiastici avvenute in Francia nel settembre I792. (cfr. Settembrizzare, «Ospizio», XXII).

La definizione è corredata da un esempio, che risale al I850:

La morte di Luigi XVI era già, agli occhi degli oratori della sinistra, dei settembriani [...] la necessaria sanzione del trionfo della rivoluzione. (Memorie autografe di Robespierre, volgarizzamento da M. Guitera de Bozzi, Firenze, presso Antonio Tozzeti, I850, p. 138)

In rete il termine è poco attestato: nei pochi esempi rintracciati è comunque sempre usato come 
aggettivo col significato 'di settembre'.

- settembrile

L'aggettivo settembrile 'di settembre', sinonimo di settembrino, è registrato esclusivamente dal GRADIT, che lo marca come termine di basso uso, e dal GDLI, che lo considera una voce letteraria:

Nella tiepida notte settembrile / si scioglie la lussuria del giardino / e dorme nella vasca un amorino / sull'acqua con dolcezza femminile. (Giuseppe Villaroel, Nella tiepida notte, in Giuseppe Villaroel: cinquant'anni di attivitá letteraria, a cura del Comune di Catania, Firenze, Leo S. Olschki, 1962, p. 39)

- settembrino

Il termine settembrino, registrato dalla maggior parte dei dizionari sincronici, è usato come aggettivo col significato 'di settembre, relativo al mese di settembre' (pioggia settembrina, sole settembrino, clima settembrino, lana settembrina):

Vill'Amarena! Dolce la tua casa / in quella grande pace settembrina! (Guido Gozzano, Opere, a cura di Carlo Calcaterra e Alberto De Marchi, Milano, Garzanti, 1956, p. I27)

Il sostantivo settembrino appartiene invece all'ambito della botanica e indica il nome comune di alcune piante del genere Aster.

Il termine fa inoltre parte della tradizione proverbiale, soprattutto toscana. Il Dizionario dei proverbi italiani di Schwamenthal e Straniero (I99I), l'Atlante Paremiologico Italiano a cura di Temistocle Franceschi (2000), il Dizionario dei proverbi di Boggione e Massobrio (2004) e il Dizionario dei proverbi italiani di Carlo Lapucci (2006) citano i seguenti proverbi: Alla luna settembrina, sette lune se le inchina (la luna di settembre ci fa prevedere le sette lune, o fasi lunari, successive) e le varianti La luna settembrina, sette lune si strascina, Luna settembrina sette se ne trascina e Se piove sulla luna settembrina sette di l'acqua trascina; Aria settembrina, fresco la sera e fresco la mattina (ci si avvicina all'equinozio d'autunno; le giornate sono più corte e fresche e la notte diventa più lunga, raffreddando la terra); Luna settembrina quando t'alzi c'é la brina; La luna settembrina: o piove o vento tira; Sole settembrino prendilo in cammino (cioè, come quello di marzo, muovendoti e camminando) e la variante Il sole settembrino giova prenderlo in cammino; Acqua settembrina rovina il vino; Mese settembrino: acqua, sole e buon vino.

Settembrino e Settembrini, insieme a Settembre e Settembri, sono inoltre cognomi italiani. A proposito del cognome Settembre, si legge nel Dizionario dei cognomi italiani di Emidio De Felice (Milano, Mondadori, I992): "raro e sporadico, piu comune nel napoletano, ha alla base il nome settembre o settembrino, dato nel passato a bambini nati nel mese di settembre".

\section{Cita come:}

Lucia Francalanci, Derivati dei nomi dei mesi (Settembre), "Italiano digitale", XVIII, 2021/3 (lugliosettembre)

DOI: 10.35948/2532-9006/2021.11606

Copyright 2021 Accademia della Crusca

Pubblicato con licenza creative commons CC BY-NC-ND 